\title{
INOVAÇÃO EM MICRO E PEQUENAS EMPRESAS POR MEIO DO SERVIÇO BRASILEIRO DE RESPOSTAS TÉCNICAS
}

\author{
Ricardo Augusto Bonotto Barboza \\ Doutor em Alimentos e Nutrição pela Universidade Estadual Paulista Júlio de Mesquita Filho - \\ UNESP \\ Professor do Centro Universitário de Araraquara - UNIARA \\ bonotto@sirtunesp.com.br (Brasil)
}

\section{Sérgio Azevedo Fonseca}

Doutor em Administração pela Universidade de São Paulo -USP

Professor da Universidade Estadual Paulista Júlio de Mesquita Filho - UNESP

saf@fclar.unesp.br (Brasil)

\section{Geralda Cristina Freitas Ramalheiro}

Bacharel em Administração Pública pela Universidade Estadual Paulista Júlio de Mesquita Filho UNESP

geraldar@sirtunesp.com.br (Brasil)

\section{RESUMO}

Este artigo relata os resultados de uma pesquisa realizada com norte em dois objetivos: o primeiro, traçar o perfil, institucional, estrutural e operacional de um sistema de apoio a inovações de baixa complexidade para empreendimentos de pequeno porte (SBRT), organizado no formato de rede nacional de agentes acadêmicos, tecnológicos e representativos do meio empresarial; e o segundo (e principal) estabelecer um paralelismo entre a estratégia predominante do SBRT e a estratégia, alternativa e inovadora, adotada por um dos agentes da rede. A pesquisa, qualitativa, exploratória e delineada como um estudo survey, revelou que a estratégia central da rede é a de atendimento a demandas espontâneas, restringindo-se à prestação de serviços de elaboração de notas técnicas. Já a instituição cujas atividades foram avaliadas buscou diferenciar suas atividades, adotando uma estratégia de indução de demandas, que vai além da simples elaboração de notas técnicas e passa a atuar como efetivo agente de inovações.

Palavras-Chaves: Resposta técnica; Inovações de baixa complexidade; Empreendimentos de pequeno porte; Gestão do conhecimento; Gestão da inovação. 


\section{INTRODUÇÃO}

O conceito de inovação tem suscitado, nas últimas décadas, intenso debate nos meios acadêmicos, políticos e empresariais. As razões dessa crescente atenção vão além dos profundos impactos que as inovações, especialmente as tecnológicas, têm causado e vêm causando nas empresas, na economia, no Estado e na sociedade como um todo. Grande parte da literatura sobre o tema (Castelli \& Wilkinson, 2013; Quandt, 2012; Vieira Filho \& Silveira, 2012) atribui uma conotação econômica e associa a ideia de inovação à noção de mudança tecnológica, criando vínculo estreito entre inovação e inovação tecnológica o que por si só intensifica e torna mais complexo o percurso para a inovação. Isso se deve a dois fatores, um de caráter teórico e outro contextual. Do lado teórico está a herança Schumpeteriana, que fornece parcela substancial dos alicerces para o debate acerca do tema; do lado contextual estão os impactos econômicos e produtivos resultantes das mudanças tecnológicas recentes, que muitas vezes singularizam a inovação apenas como inovação em produto, legando a um plano secundário os demais tipos de inovação contemplados pela literatura.

Quando se analisa o processo inovativo em micro e pequenas empresas (MPEs) evidencia-se que as dificuldades enfrentadas pelas mesmas acabam sendo ainda mais acentuadas do que no caso das médias e grandes empresas. Dificuldades que, de acordo com a literatura, são passíveis de serem superadas com o aporte e a transferência de tecnologias e conhecimentos já disponíveis nas universidades (Bodas Freitas, Geuna, \& Rossi, 2013; Kalar \& Antoncic, 2015; Landry, Amara, Cloutier, \& Halilem, 2013; McKelvey, Zaring, \& Ljungberg, 2014; Theodorakopoulos, Snchez Preciado, \& Bennett, 2012; Tödtling, Lehner, \& Kaufmann, 2009). Contudo, para que a superação ocorra devem estar disponíveis mecanismos que facilitem esse aporte e transferência, contribuindo para a redução do histórico e crônico distanciamento entre os dois campos (o da universidade e o dos empreendimentos de pequeno porte). São mecanismos capazes de funcionarem como dutos transmissores dos conhecimentos armazenados e produzidos na universidade, com potencial de gerarem inovações nas MPE. Em virtude, no entanto, do grande número de empreendimentos de pequeno porte existentes no Brasil, estimado em mais de 10 milhões (Sebrae, 2015), da acentuada dispersão geográfica desses empreendimentos e da grande diversidade tipológica e setorial inerente aos mesmos, tornar-se-ia pouco efetiva e de alto custo a criação e a operação de unidades individuais de interface, vinculadas a cada universidade do país, com potencial de cooperação com os universos locais e regionais de MPE.

A percepção desses desafios motivou o Ministério de Ciência Tecnologia e Inovação (MCTI) a criar em 2002, em parceria com Serviço Brasileiro de Apoio a Micro e Pequenas Empresa (SEBRAE),

Revista de Administração e Inovação, São Paulo, v. 12, n.3 p. 330-349, jul./set. 2015. 
o Serviço Brasileiro de Respostas Técnicas (SBRT), abrangendo uma rede de instituições, com ramificação por todo o território brasileiro, que passaram a operar um conjunto de ferramentas de gestão do conhecimento, tratadas como notas técnicas, potencialmente indutoras de inovações de baixa complexidade, tendo como públicos-alvo os novos empreendedores e os empreendimentos de pequeno porte. Trata-se de uma rede cuja estratégia de operações está essencialmente orientada para o atendimento a demandas espontâneas, sem o correspondente monitoramento para a coleta de evidências relativas às potenciais ou efetivas inovações geradas. $\mathrm{O}$ caráter inovador da rede - sua concepção, seu formato e sua estratégia de operações são inéditos em âmbito internacional - aliado à escassez da literatura focada nesse objeto particular, acrescido da ausência de evidências quanto às efetivas contribuições das notas técnicas para a geração de inovações, foram os fatores que motivaram a realização da pesquisa cujos resultados são relatados e interpretados neste artigo.

Este artigo relata os resultados de uma pesquisa realizada com norte em dois objetivos: o primeiro, traçar o perfil, institucional, estrutural e operacional de um sistema de apoio a inovações de baixa complexidade para empreendimentos de pequeno porte (SBRT), organizado no formato de rede nacional de agentes acadêmicos, tecnológicos e representativos do meio empresarial; e o segundo (e principal) estabelecer um paralelismo entre a estratégia predominante do SBRT e a estratégia, alternativa e inovadora, adotada por um dos agentes da rede. Trata-se de objetivos que dialogam, estreita e proximamente, com a questão norteadora da pesquisa, expressa como: Quais as virtudes e limitações da estratégia do SBRT, de atendimento a demandas espontâneas, vis-à-vis a estratégia de indução de demandas, adotada por um dos seus agentes de respostas técnicas?

O texto está estruturado em sete seções. Esta primeira, introdutória. A segunda, em que é abordado o conceito de inovação, com base na literatura. Na terceira são tratadas algumas das especificidades da inovação em empreendimentos de pequeno porte. A quarta aborda o histórico, a composição e as características do SBRT. A quinta trata da estratégia metodológica utilizada na pesquisa. A sexta compreende os resultados da pesquisa. Finalmente na última são apresentadas as conclusões e são tecidas as considerações finais.

\section{BREVE DISCUSSÃO SOBRE INOVAÇÃO}

A inovação vem sendo considerada, crescentemente, fator crítico para a competitividade e a sobrevivência das empresas em seus ambientes, setoriais e de mercado, bem como para a credibilidade das organizações não empresariais nos contextos dos meios sociais em que atuam. No âmbito

Revista de Administração e Inovação, São Paulo, v. 12, n.3 p. 330-349, jul./set. 2015. 
macroeconômico, é um fator propulsor das políticas de geração de emprego e renda, capaz de reduzir as externalidades negativas provenientes do sistema produtivo (McGuirk, Lenihan, \& Hart, 2015; Schumpeter, 1934, 1961).

Entende-se inovação como uma ideia que, incorporada no âmbito das organizações, em atividades produtivas, operacionais ou de gestão, propicia melhorias e/ou mudanças, seja em processos internos, seja nos resultados finais das operações organizacionais. Assim, a inovação é vista como a realização de novas combinações na forma de um novo produto, um novo método de produção, a abertura de um novo mercado, uma nova fonte de fornecimento de materiais ou produtos semimanufaturados e, finalmente, a nova organização de uma indústria (Schumpeter, 1961). Velu (2014) ressalta que a inovação tecnológica pode ser materializada em mudanças nas concepções de produtos, processos e modelo de negócios, sendo que a inovação de produto implica a criação de diferentes vantagens para o cliente em relação aos produtos anteriores que estavam disponíveis no mercado. As inovações em processos, por sua vez, envolvem melhorias na produção ou nos processos de distribuição e são capazes de reduzir os custos médios e aumentar as margens de lucro. Por fim, a inovação no modelo de negócio envolve a descoberta e adoção de uma nova forma de disponibilizar valor, capturar valor e/ou criar valor para uma empresa ou para o seu cliente (Velu, 2014).

Com o propósito de esboçar um esquema analítico que venha a oferecer suporte para a interpretação dos resultados da pesquisa, é pertinente abordar o conceito de inovação, ainda que de forma breve e sintética, por meio de algumas categorias tipológicas.

Na primeira categoria, relativa à intensidade e profundidade dos impactos, as inovações são, desde Schumpeter, classificadas como incrementais ou radicais (Radas \& Božić, 2009; Velu, 2014). A inovação incremental é a introdução de mudanças relativamente marginais ou de menor escala na concepção de um produto ou de um processo existente. Por outro lado, a inovação radical é baseada em desvios significativos em relação ao design existente e à concepção do produto. Esse último tipo de inovação, potencialmente, abre novas aplicações e novos mercados, requer mudanças significativas nas rotinas organizacionais e nos processos das empresas criadoras.

A segunda dimensão é a relativa à origem institucional das inovações. Velu (2014) destaca que as inovações podem ser obtidas por meio de processos internos (logo fechados e que são desenvolvidos no interior das organizações) ou por meio de processos abertos em que se destaca a interação e a troca de informações entre diferentes organizações. A literatura de inovação estratégica reconhece, cada vez mais, que a combinação de fontes de conhecimento interno e externo é um elemento-chave de uma estratégia de inovação bem-sucedida (Love, Roper, \& Vahter, 2014). 
A terceira categoria analítica diz respeito aos processos por meio dos quais as inovações são geradas e difundidas. Ganha destaque aqui o conceito de inovação aberta, que passa a ganhar reconhecimento e importância crescentes com o advento da globalização e das implícitas sinergias interativas (Chesbrough, 2003; Love et al., 2014; McKelvey et al., 2014; Tödtling et al., 2009; Velu, 2014). As inovações passam a ser vistas, de forma crescente, como o resultado de um processo integrado e articulado de geração de conhecimento, difusão e aplicação (Tödtling et al., 2009). Para esses últimos autores as inovações estão ocorrendo dentro de uma complexa rede de relações formais e informais, estáticas e dinâmicas. Os autores observam, também, que as interações e as diferentes fontes de conhecimento usadas no processo inovativo influenciam no tipo de inovação gerada. Inovações tipicamente tecnológicas, com maiores graus de complexidade, se desenham a partir de novos conhecimentos científicos que são gerados no interior das universidades e organizações de pesquisa. Supõe-se, frequentemente, que a troca desse tipo de conhecimento exige interações pessoais e intensas, o que favorece os níveis locais e regionais. Por outro lado, as inovações de baixa complexidade tendem a ocorrer mais por interações entre parceiros do setor empresarial, muitas vezes situados em níveis espaciais superiores, fora da região (Tödtling et al., 2009).

A quarta dimensão abrange as competências para a inovação. Love et al. (2014) e D’Este et al. (2012) defendem que a inovação depende crucialmente da capacidade das empresas de absorverem conhecimentos externos, combinando-os com seus próprios conhecimentos, tácitos ou explícitos. Por consequência, um elemento importante neste processo é a identificação e o aproveitamento de conhecimentos complementares entre diferentes atividades dentro e fora dos limites da empresa. Entre as competências internas para a inovação destacam-se: a presença de um corpo de profissionais especializados e qualificados; a forte liderança dos responsáveis pela organização; a existência de estratégias e planejamento para o futuro; e as atividades de pesquisa e desenvolvimento (D'Este et al., 2012; Radas \& Božić, 2009). São competências necessárias para o diálogo e a colaboração com outras empresas, para a manutenção de relações de cooperação para a pesquisa e a transferência de tecnologia com universidades e centros de pesquisa e para a captação de recursos financeiros ou demais tipos de apoios governamentais (D’Este et al., 2012; Radas \& Božić, 2009).

A quinta dimensão analítica é a que diz respeito ao porte das empresas. O mundo empresarial é marcado pela grande heterogeneidade. Empresas possuem características diversas em relação aos recursos disponíveis, às competências para a inovação e às capacidades para criarem valor a partir dos conhecimentos gerados (D’Este et al., 2012; Landry et al., 2013). Isso implica evidentes discrepâncias nas propensões relativas para inovar entre empresas de diferentes portes.

Revista de Administração e Inovação, São Paulo, v. 12, n.3 p. 330-349, jul./set. 2015. 
Inovação em micro e pequenas empresas por meio do serviço brasileiro de respostas técnicas

Quando contextualizada a inovação para o público alvo deste estudo, ou seja, Micro e Pequenas Empresas (MPEs), características peculiares das mesmas condicionam e orientam o percurso e a tipologia de inovações (Barge-Gil, 2010; Doh \& Kim, 2014; Huizingh, 2011; McGuirk et al., 2015; McKelvey et al., 2014; Radas \& Božić, 2009; Solomon, Bryant, May, \& Perry, 2013; Spithoven, Clarysse, \& Knockaert, 2011). A próxima seção irá discutir as particularidades da inovação no contexto das micro e pequenas empresas, com especial atenção às dificuldades de operacionalização.

\section{BREVE DISCUSSÃO SOBRE INOVAÇÃO EM EMPREENDIMENTOS DE PEQUENO PORTE}

A inovação no contexto dos empreendimentos de pequeno porte tem sido objeto de curiosidade, atenção e pesquisa nas últimas décadas (Amato Neto \& Olave, 2005; Barge-Gil, 2010; Doh \& Kim, 2014; Freel, 2000; Huizingh, 2011; McGuirk et al., 2015; McKelvey et al., 2014; Mundt \& Bignetti, 1992; Oakey, Rothwell, \& Coope, 1988; Radas \& Božić, 2009; Solomon et al., 2013; Spithoven et al., 2011). A literatura revela que a inovação representa, ora fator de surgimento de novos empreendimentos na fronteira do conhecimento (no caso, as pequenas empresas de base tecnológica) ora fator de sobrevivência para empreendimentos já existentes (sobretudo para as pequenas empresas atuantes em setores mais tradicionais da economia, com tecnologias maduras). As primeiras mais envolvidas em atividades de pesquisa e desenvolvimento, as últimas adotando inovações de menor complexidade (Doh \& Kim, 2014; McGuirk et al., 2015).

Nota-se que os tipos de inovação mais frequentes adotados por empreendimentos de pequeno porte são qualificados por autores como Grimpe e Sofka (2009), Heidenreich (2009), Kirner, Kinkel \& Jaeger (2009), Nunes, Serrasqueiro e Leitão (2012) e Barge-Gil (2010) como inovações não tecnológicas ou inovações de baixa tecnologia (low-tech innovations). A segunda característica das inovações em pequenas empresas, defendida por autores como Grimpe e Sofka (2009), Heidenreich (2009), Vrgovic et al. (2014), Tödtling, Lehner e Kaufmann (2009), entre outros, é a de que o percurso pelo qual a extensa maioria incorpora inovações seria enquadrado no conceito de inovações abertas, tal como definido por Chesbrough (2003). Radas e Božić (2009) defendem que os fatores que influenciam a inovação nas micro e pequenas empresas podem ser divididos em internos e externos, os primeiros referidos a características e políticas das MPE, os últimos às oportunidades que as MPE podem aproveitar do ambiente. Doh e Kim (2014) notaram que, no contexto das pequenas empresas, a inovação em produto é vista como o resultado do investimento em competitividade por diferenciação e

Revista de Administração e Inovação, São Paulo, v. 12, n.3 p. 330-349, jul./set. 2015. 
como estratégia de expansão do mercado e que as inovações em processos surgem como uma estratégia de competitividade por preços e como um caminho para a eficiência.

Em relação às indústrias tradicionais, e que são predominantemente caracterizadas pela presença de micro e pequenas empresas, constatou-se que as mesmas apresentam limitações para executar atividades de P\&D e para gerar inovações (Spithoven et al., 2011). Como evidenciou BargeGil (2010) as empresas fora dos setores de alta tecnologia, as empresas menores e, em certa medida, as empresas com baixa intensidade $\mathrm{P} \& \mathrm{D}$, inovam, frequentemente, por meio da cooperação, isso porque o número e a qualificação dos funcionários de muitas dessas empresas caem abaixo de uma massa crítica necessária (Spithoven et al., 2011). Os resultados de Mcguirk, Lenihan e Hart (2015) revelam que os funcionários de pequenas empresas que participam de treinamento têm maiores probabilidades de participarem de atividades inovativas. Outro fator propulsor da inovação em pequenas são as participações em redes (Doh \& Kim, 2014).

No que tange às dificuldades das MPE para inovar, a literatura (Amato Neto \& Olave, 2005; Barge-Gil, 2010; Doh \& Kim, 2014; Freel, 2000; Huizingh, 2011; McGuirk et al., 2015; McKelvey et al., 2014; Mundt \& Bignetti, 1992; Oakey et al., 1988; Radas \& Božić, 2009; Solomon et al., 2013; Spithoven et al., 2011) aponta a existência de um conjunto de fatores restritivos, resultantes das circunstâncias de:

- Operarem em segmentos de mercado relativamente estáveis, com produtos/serviços padronizados, alto grau de homogeneidade e valor agregado relativamente baixo;

- Ofertarem produtos e serviços de baixa complexidade tecnológica, estimulando a concorrência nos mercados;

- Utilizarem processos produtivos relativamente rígidos;

- Possuírem uma administração tradicional (paternalista, autoritária), centrados em geral nos proprietários, nem sempre tecnicamente qualificados, que usam alto grau de empirismo para acumular conhecimentos em suas áreas de negócio;

- Possuírem um perfil de força de trabalho predominantemente de baixa qualificação, com relações informais de trabalho se sobrepondo às profissionais;

- Adotarem processos de inovação essencialmente empíricos, pontuais e assistemáticos, raramente apoiados em atividades de $\mathrm{P} \& \mathrm{D}$ formais;

- Utilizarem, na maior parte das vezes, componentes tecnológicos gerados externamente, agregados por fornecedores de materiais e equipamentos;

- Possuírem uma cultura restritiva a percursos de inovações contínuas, por apresentarem

Revista de Administração e Inovação, São Paulo, v. 12, n.3 p. 330-349, jul./set. 2015. 
foco em resultados de curto prazo, falta de visão de longo prazo e temor ao risco e às incertezas próprias do processo de inovação;

- Não possuírem cultura para desenvolver projetos em parceria com universidades e instituições de pesquisa;

- Por fim, se depararem com a regulamentação excessiva e menor controle sobre o ambiente externo.

Esse conjunto de fatores, inibidores de processos e atividades passíveis de criarem ambientes favoráveis à inovação, no contexto das micro e pequenas empresas, dos empreendimentos informais e dos empreendedores de baixa cultura e formação tecnológica, oferecem o desafio, à universidade, ao governo e aos próprios empreendimentos, para conceberem, implementarem e operacionalizarem instrumentos e mecanismos indutores de inovações.

Ressalta-se que as micro e pequenas empresas mantém fortes laços com suas localidades, integrando-se regionalmente, criando dependências para com as especificidades de suas regiões e das políticas públicas existentes nos planos locais (Radas \& Božić, 2009). Doh e Kim (2014) reforçam essa linha de raciocínio afirmando que muitos dos problemas enfrentados pelas MPE tradicionais demandam, dos governos locais, iniciativas políticas para melhorar o acesso das MPE ao financiamento de infraestruturas e de informação necessárias ao processo de difusão e aplicação de conhecimentos. Devem também criar marcos regulatórios, legais e financeiros favoráveis ao empreendedorismo, e à geração de inovações.

No Brasil existem alguns programas voltados para o apoio às micro e pequenas empresas, entre eles destacam-se: o Programa de Apoio Tecnológico às Micro e Pequenas Empresas - PATME; o Programa de Extensão Tecnológica Industrial - PET; o programa FAPESP de Pesquisa Inovativa em Pequenas Empresas - PIPE; os instrumentos oferecidos pelo Serviço Brasileiro de Apoio a Micro e Pequena Empresa - SEBRAE, destacando-se o programa de apoio as entidades de pesquisa através de desenvolvimento, conhecimento e aplicação de tecnologias - Programa SEBRAETec, do SEBRAESP. São programas que, no entanto, possuem alcance restrito, demandam a mobilização de competências técnicas especializadas (próprias das empresas ou contratadas externamente), pressupõem a elaboração de projetos técnicos com tempo relativamente elevado de maturação e se orientam para inovações de média para alta complexidade. São características que, pela sua natureza, são limitantes, quando não impeditivas, ao acesso dos empreendimentos de pequeno porte a esses programas. 
A constatação dessas restrições, aliada à percepção da importância de sua superação, levou a Universidade de São Paulo - USP a criar, em 1991, um programa de atendimento a demandas por conhecimentos e informações técnicas de baixa complexidade, especialmente voltado para um público constituído por empreendimentos de pequeno porte situados nos setores tradicionais da economia, detentores de tecnologias, processos e produtos considerados maduros. O programa, conhecido como Disque-Tecnologia - DT/USP, ganhou reconhecimento e, em poucos anos, teve o seu conceito difundido e incorporado por outras instituições no Brasil. A disseminação do modelo propiciou a criação, em 2002, de uma rede nacional de instituições operando com respostas técnicas, que passou a ser conhecida como Serviço Brasileiro de Respostas Técnicas - SBRT.

A composição, a estrutura, a sistemática operacional e o breve descritivo dos serviços prestados são aspectos tratados na próxima seção.

\section{O SERVIÇO BRASILEIRO DE RESPOSTAS TÉCNICAS (SBRT)}

O SBRT passou a ser formado, a partir de 2010, por nove instituições: Serviço Nacional de Aprendizagem Industrial do Rio Grande do Sul - SENAI/RS; Instituto Tecnológico do Paraná TECPAR; Universidade de São Paulo - USP; Universidade Estadual Paulista - UNESP; Rede de Tecnologia do Rio de Janeiro - REDETEC; Centro de Tecnologia de Minas Gerais - CETEC; Universidade de Brasília - UNB; Serviço Nacional de Aprendizagem Industrial do Amazonas SENAI/AM; Rede de Tecnologia da Bahia - RETEC. A distribuição regional do atendimento dessas instituições é ilustrada na figura 1. 


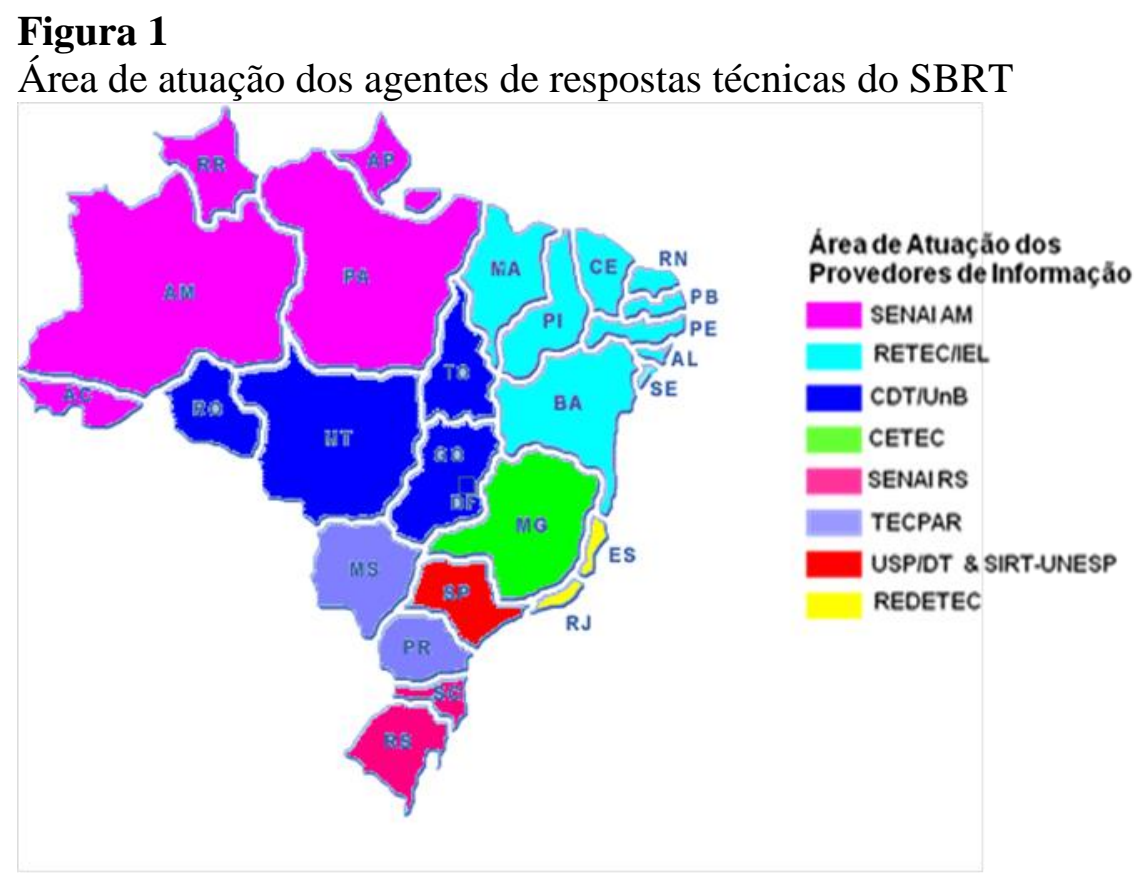

Fonte: Serviço Brasileiro de Respostas Técnicas SBRT (2012)

Duas características principais são definidoras da estratégia nuclear do SBRT: o atendimento a demandas espontâneas, implicando que os agentes de respostas vinculados à rede são acionados apenas quando uma demanda é postada no sistema; e o foco na gestão do conhecimento, denotando que, embora o sistema tenha sido concebido como instrumento de apoio a inovações, suas atividades são essencialmente orientadas para as operações de busca, elaboração, sistematização, armazenagem e disponibilização de informações. A materialização desta estratégia é viabilizada pela oferta de serviços de apoio a informação.

A tipologia dos serviços prestados pelo SBRT é composta por quatro tipos de notas técnicas: a Resposta Técnica (RT), a Resposta Técnica Complementar (RTC), a Resposta Referencial (RR) e o Dossiê Técnico (DT). Em conformidade com as instruções de trabalho do SBRT (Fonseca \& Barboza, 2014), esses são documentos que possuem as características explicitadas no quadro 1.

\section{Quadro 1}

Descrição da tipologia de serviços ofertados pelo SBRT

\begin{tabular}{|c|l|}
\hline $\begin{array}{c}\text { Tipos de notas } \\
\text { técnicas }\end{array}$ & \multicolumn{1}{c|}{ Definições - caracterizações } \\
\hline $\begin{array}{c}\text { Resposta Técnica } \\
\text { (RT) }\end{array}$ & $\begin{array}{l}\text { É compreendida como um conjunto de informações técnicas de baixa } \\
\text { complexidade, relativas à produção de um bem industrial, à execução de um } \\
\text { serviço técnico especializado, ao cultivo e criação de produtos agropecuários, } \\
\text { que envolvam aspectos tecnológicos e que visem à melhoria da qualidade, ao }\end{array}$ \\
\hline
\end{tabular}

Revista de Administração e Inovação, São Paulo, v. 12, n.3 p. 330-349, jul./set. 2015. 


\begin{tabular}{|c|l|}
\hline & $\begin{array}{l}\text { aumento de produtividade e à solução de problemas técnicos/operacionais de } \\
\text { interesse das micro e pequenas empresas, bem como para início e } \\
\text { diversificação de negócios. Essas informaçães de caráter referencial são } \\
\text { capazes de subsidiar e contribuir com soluções a dúvidas e problemas } \\
\text { empresariais e ou tomada de decisões (não muito complexas). }\end{array}$ \\
\hline $\begin{array}{c}\text { Resposta Técnica } \\
\text { Complementar } \\
\text { (RTC) }\end{array}$ & $\begin{array}{l}\text { São notas técnicas que informam sobre normalização, regulamentação, } \\
\text { legislação e fornecedores de produtos acabados e serviços especializados no } \\
\text { auxílio do processo produtivo da empresa. }\end{array}$ \\
\hline Resposta Referencial \\
(RR) & $\begin{array}{l}\text { É uma nota técnica que esclarece ou orienta o usuário para a obtenção da } \\
\text { informação solicitada, seja por consultoria tecnológica ou apoio à gestão, } \\
\text { indicando a instituição onde ele poderá receber o atendimento. }\end{array}$ \\
\hline Dossiê Técnico (DT) & $\begin{array}{l}\text { É uma nota técnica de mais alta complexidade, elaborada com o propósito de } \\
\text { oferecer orientações para a produção de um bem industrial, a execução de um } \\
\text { serviço técnico especializado, o cultivo e criação de produtos agropecuários, } \\
\text { envolvendo aspectos tecnológicos e visando à melhoria da qualidade, ao } \\
\text { aumento de produtividade e à solução de problemas técnicos/operacionais. As } \\
\text { demandas são identificadas pelos agentes integrantes da rede, buscando suprir } \\
\text { necessidades locais e regionais onde estão inseridas as instituições. Os DTs } \\
\text { têm que ser previamente aprovados pelo SBRT. }\end{array}$ \\
\hline
\end{tabular}

Fonte: (Fonseca \& Barboza, 2014)

O fluxo de atendimento do SBRT é composto pelas seguintes etapas: primeiro o usuário acessa o site do SBRT, se cadastra e lê atentamente o termo de uso; como passo seguinte, consulta o Banco de Dossiês e Respostas Técnicas do SBRT, onde poderá encontrar respostas para sua demanda; caso não encontre a solução esperada, submete nova demanda. A demanda é encaminhada ao agente regional da rede que será responsável por elaborar a resposta. Na sequência, a resposta é publicada no site e o usuário recebe um e-mail informando que ela está pronta e que pode ser consultada a qualqer momento.

O banco de dados do SBRT continha, em 2014, mais de 21 mil notas técnicas classificadas por palavras-chave, elaboradas pelos nove agentes da rede e é de livre acesso a todos os cadastrados no site. É importante ressaltar que, caso a demanda não se inclua em nenhum dos escopos instruídos normativamente pelo SBRT, o usuário recebe a informação na forma de Resposta Referencial (RR), pela qual é orientado a buscar outras fontes de consulta.

O que vem sendo constatado ao longo da história do SBRT é que, embora a rede seja provedora de informações passíveis de gerarem inovações, não tem a sua estratégia direcionada ao campo da gestão da inovação. A percepção dessa limitação norteou um dos agentes vinculados à rede - o Sistema Integrado de Respostas Técnicas da Unesp, SIRT/Unesp - a buscar a diferenciação das suas atividades, passando a atuar em duas novas frentes: a de indução de demandas e a de assistência aos usuários. Isso propiciou uma aproximação ao campo da gestão da inovação.

O SIRT/Unesp nasceu com uma proposta inovadora, de atuação proativa, visando à prospecção e à indução de demandas, guardando afinidade com a ideia de criação de um instrumento de política 
pública de apoio ao desenvolvimento local. A estratégia do SIRT/Unesp, complementar à do SBRT, passou a ser a de induzir demandas de segmentos homogêneos e cadeias curtas, abrangendo empreendimentos de pequeno porte, na perspectiva de apoiar políticas de desenvolvimento local por meio da geração de inovações de baixa complexidade.

Como primeiro passo para testar o potencial e a efetividade dessa estratégia de atuação o SIRT buscou estabelecer parcerias com incubadoras de empresas situadas em municípios localizados na região central do interior do Estado de São Paulo. O primeiro município contemplado com a parceria foi Araraquara, escolha esta justificada pela proximidade física. Essa decisão, de testar a estratégia em incubadoras, embora reconhecendo que as mesmas possam não abranger segmentos homogêneos ou cadeias curtas, pautou-se na opção por um tipo de ambiente com concentração espacial de empreendimentos de pequeno porte, potencialmente propensos à adoção de inovações.

A pesquisa, cuja metodologia é brevemente descrita na próxima seção, buscou apurar e avaliar os primeiros resultados dessa estratégia, tendo como referencial comparativo a estratégia dominante do SBRT, de atendimento a demandas espontâneas.

\section{METODOLOGIA}

Para o alcance do objetivo propugnado, realizou-se uma pesquisa descritiva. Isso porque, como lembram Yin (2010) e Selltiz et al. (1987), as pesquisas descritivas visam: a) apresentar, de forma precisa, as características de uma situação, um grupo, um indivíduo ou uma organização específica; b) descobrir ou verificar a existência de relações entre variáveis (no caso, entre variáveis, de um lado, do grupo de usuários por demandas induzidas, ora chamados usuários SIRT e, de outro, do grupo - de controle - de usuários por demandas espontâneas, ora chamados usuários SBRT; c) identificar resultados, fatores e situações passíveis de serem generalizáveis. Além de descritiva, a pesquisa também deve ser considerada exploratória, tanto em virtude da impossibilidade de generalização dos seus resultados, quanto pelo caráter relativamente desconhecido do fenômeno sob investigação e ainda pelo estágio marcadamente preliminar da pesquisa. Para a definição do delineamento, recorreu-se à triangulação de métodos, ou seja, uma "estratégia de pesquisa que se apoiou em métodos científicos testados e consagrados, servindo e adequando-se a determinadas realidades, com fundamentos interdisciplinares" (Minayo, Assis, \& Souza, 2014, p. 71). Inicialmente efetivou-se a pesquisa documental, que recorreu a fontes mais diversificadas e dispersas, sem tratamento analítico, tais como a base de dados do SBRT. O passo seguinte foi a pesquisa survey, que buscou informações diretamente 
com um grupo de interesse a respeito dos dados que se desejava obter, no caso os usuários do SIRT. Para tanto foi utilizado um questionário semiestruturado como instrumento de pesquisa.

Para a análise dos dados foi elaborada uma matriz contendo dois grupos de dados: o primeiro, relativo às notas técnicas elaboradas pelo SIRT/Unesp com base nas demandas espontâneas recebidas; o segundo, pelas notas técnicas elaboradas a partir de demandas induzidas. O período compreendido foi o de fevereiro de 2011 a dezembro de 2012.

Foram consideradas espontâneas as demandas que haviam sido cadastradas no site pelo próprio empreendedor, sem nenhuma intervenção dos mediadores do SIRT/Unesp. As demandas induzidas, por sua vez, foram aquelas oriundas da atuação direta dos mediadores junto às empresas da incubadora. Apurou-se que, no período, foram elaboradas 999 notas técnicas, com atendimento a 697 usuários de todo o Estado de São Paulo. Desse total, 140 notas foram geradas por indução na incubadora de empresas de Araraquara por meio do atendimento in loco a 10 empreendedores incubados. A tabela 1 retrata esses resultados

\section{Tabela 1}

Números de notas técnicas produzidas e usuários atendidos pelo agente SIRT/Unesp no período de fevereiro de 2011 a dezembro de 2012

\begin{tabular}{|l|l|l|}
\hline Tipo de demandas & $\mathbf{N}^{\circ}$ - Notas Técnicas & $\mathbf{N}^{\circ}$ de usuários atendidos \\
\hline Espontânea & 859 & 687 \\
\hline Induzida & 140 & 10 \\
\hline Total & $\mathbf{9 9 9}$ & $\mathbf{6 9 7}$ \\
\hline
\end{tabular}

Fonte: Elaboração própria (2013)

Para a análise dos dados contidos na matriz elaborada foram adotados os seguintes critérios:

1. Foram investigados apenas os usuários que demandaram RT e RTC. Tal fato justifica-se diante dos seguintes fatores: os dossiês técnicos não haviam sido elaborados a partir de demandas, mas por iniciativa da própria coordenação do SIRT; as RR geralmente remetem a duvidas de gestão ou de alta complexidade, não se enquadrando no escopo do SBRT.

2. Foram consideradas apenas notas técnicas elaboradas para empreendedores cujo perfil estivesse completo e atualizado. Tal decisão se justificou diante da necessidade de se estabelecer contatos com os usuários das notas técnicas e as imprecisões nos registros impossibilitariam tais contatos. 
Com base nesses critérios foram descartadas 571 notas técnicas (sendo 249 referentes a Respostas Referenciais (RR) e outras 322 referentes a registros imprecisos). Passaram a ser consideradas, como válidas para a pesquisa, 428 notas técnicas (42,84\% do total), sendo 140 relativas a demandas induzidas na incubadora de empresas de Araraquara e as outras 288 referentes a demandas espontâneas.

Importante observar que o SBRT permite que um mesmo empreendedor poste mais de uma demanda no sistema, implicando que o mesmo cadastro pode abranger várias notas técnicas. Procedida a essa filtragem o número de usuários da amostra ficou em 240 (34,43\% do total).

Identificada a amostra que seria investigada (notas técnicas e os respectivos usuários), procedeu-se à coleta de dados, entre os meses de janeiro e fevereiro de 2013, por meio de um roteiro semiestruturado. O roteiro foi segmentado em quatro blocos: o primeiro, buscando avaliar se houve aplicação, por parte dos usuários, dos conteúdos das notas técnicas. No segundo, buscou-se apurar, nas percepções dos usuários, quais foram as contribuições das notas técnicas para a geração (ou não) de inovações. No terceiro bloco, procurou-se avaliar os tipos de inovações geradas, por nota técnica. O quarto bloco esteve voltado para avaliar as eventuais contribuições do SIRT para alterar a cultura empreendedora em prol da inovação.

Realizada a aplicação do questionário, procedeu-se à análise e à tabulação dos dados. A próxima seção apresenta os resultados obtidos.

\section{RESULTADOS E DISCUSSÕES}

A pesquisa revelou, na primeira das quatro partes em que foi segmentada, que cerca de $95 \%$ das demandas espontâneas tiveram, de alguma forma, o seu conteúdo aplicado. Para as demandas induzidas esse percentual ficou em $90 \%$ (tabela 2).

\section{Tabela 2}

Ocorrência da aplicação do conteúdo das notas técnicas no empreendimento

\begin{tabular}{|c|c|c|c|c|}
\hline Tipos de demanda & Aplicou & Não aplicou & Total & Frequência \\
\hline Espontânea & 273 & 15 & 288 & $94,79 \%$ \\
\hline Induzida & 127 & 13 & 140 & $90,71 \%$ \\
\hline Total & $\mathbf{4 0 0}$ & $\mathbf{2 8}$ & $\mathbf{4 2 8}$ & $\mathbf{9 3 , 4 5 \%}$ \\
\hline
\end{tabular}

Fonte: Elaboração própria (2013) 
$\mathrm{Na}$ avaliação a respeito das contribuições das notas técnicas para a geração de inovações nos contextos dos empreendimentos dos usuários, apurou-se que, nos casos das demandas induzidas, os resultados positivos foram em 62,86\% da amostra, enquanto que nos casos das demandas espontâneas, a efetividade apurada foi verificada em $37,14 \%$ da amostra (tabela 3 ).

Tabela 3

Ocorrência de inovações a partir das notas técnicas

\begin{tabular}{|c|c|c|c|}
\hline Tipos de demanda & Inovou (\%) & Não inovou (\%) & Total(\%) \\
\hline Espontânea & $107(37,15)$ & $181(62,85)$ & $288(100)$ \\
\hline Induzida & $88(62,86)$ & $52(37,14)$ & $140(100)$ \\
\hline Total & $\mathbf{1 9 5}(\mathbf{4 5 , 5 6})$ & $\mathbf{2 3 3 ( 5 4 , 4 4 )}$ & $\mathbf{4 2 8}(\mathbf{1 0 0})$ \\
\hline
\end{tabular}

Fonte: Elaboração própria (2013)

No terceiro bloco a amostra passou a ser das 195 demandas (107 espontâneas e 88 induzidas) que tinham gerado inovações. Apurou-se que as maiores proporções de inovações geradas foram as incrementais e que não houve diferenças significativas entre os dois grupos de demandas (tabela 4).

\section{Tabela 4}

Tipo de inovações geradas a partir de notas técnicas

\begin{tabular}{|l|l|l|l|l|}
\hline \multirow{2}{*}{ Tipos de Inovação } & \multicolumn{2}{|l|}{ Tipos de demanda } & \multicolumn{2}{l|}{ Frequência (\%) } \\
\cline { 2 - 5 } & Espontânea & Induzida & Espontânea & Induzida \\
\hline Novo produto no mercado & 1 & 0 & 0,9 & 0,0 \\
\hline Novo produto para a empresa & 19 & 13 & 17,8 & 14,8 \\
\hline Nova linha de produto & 9 & 15 & 8,4 & 17,0 \\
\hline Incremental & 78 & 60 & 72,9 & 68,2 \\
\hline Total & $\mathbf{1 0 7}$ & $\mathbf{8 8}$ & $\mathbf{1 0 0 , 0}$ & $\mathbf{1 0 0 , 0}$ \\
\hline
\end{tabular}

Fonte: Elaboração própria (2013)

Procedendo-se a um dos cruzamentos possíveis com os dados da pesquisa, foi possível constatar que a indução de demandas propicia, por si só, um substancial aumento nos números de demandas postadas no sistema. Essa constatação é evidenciada pelos números médios de demandas por usuário: enquanto os usuários por demandas espontâneas registraram, no período, uma média de 1,25 consultas, os usuários que tiveram suas demandas induzidas pela equipe de mediadores do SIRT submeteram uma média de 14 demandas por usuário. São dados que, no entanto, não permitem qualquer inferência quanto à efetividade de resultados, uma vez que a metodologia utilizada na pesquisa não permitiu a obtenção de dados relativos aos efeitos e consequências diretos, por demanda submetida. 


\section{CONCLUSÕES}

A primeira conclusão que se extrai da pesquisa é que os resultados que vêm sendo historicamente obtidos pela rede do SBRT ainda são tímidos apesar da grande capilaridade da rede e da simplicidade implícita na sua sistemática operacional, fator esse que poderia ser um facilitador à postagem de consultas, por quaisquer interessados. Essa conclusão é evidenciada pelo número de usuários por demandas espontâneas que foi atendido pelo SIRT, menos de 700 usuários que demandaram os serviços nos 23 meses considerados por esta pesquisa. Salienta-se que estes dados, considerando a dimensão econômica do Estado de São Paulo, as facilidades e os benefícios oferecidos pela rede, podem ser considerados irrisórios. Um indicativo das possíveis razões para esse resultado pouco satisfatório resulta de depoimentos da equipe de mediadores do SIRT que, em suas visitas a incubadoras, constatam um elevado grau de desconhecimento a respeito da existência da rede do SBRT.

Outra constatação, também extraída dos dados quantitativos da pesquisa, é que os usuários por demandas espontâneas tendem a manter um relacionamento de maior distanciamento em relação à rede do SBRT do que os usuários por demandas induzidas. Provam isso as médias de acessos aos sistemas, nos dois casos: de 1,25 consultas para o primeiro grupo para 14 no segundo grupo. A insuficiência de informações complementares para esses dados impede a formulação de inferências a respeito das razões pelas quais os usuários do primeiro grupo tendem a não acessar o sistema de forma recorrente. Esse é um indício para a continuidade da pesquisa. Um fato fica evidente, no entanto, e permite a proposição de outra conclusão, passível de ser formulada ainda neste estágio da pesquisa: o estabelecimento de relações de proximidade e confiança entre a equipe de mediadores do SBRT e os usuários do sistema tende a gerar resultados positivos em termos da formulação de demandas e da elaboração de notas técnicas. Uma inferência pode ser sugerida a partir dessa conclusão, porém necessitando de confirmação posterior: a de que o apoio mais direto e pessoal dos mediadores aos usuários da rede tende a contribuir mais efetivamente para a geração de inovações do que o apoio distante, pela simples elaboração de notas técnicas. Essa inferência encontra alguma evidência na pesquisa, quando apoiada nos dados da tabela 3.

No tocante à efetiva geração de inovações, os dados da pesquisa não oferecem evidências suficientes, passíveis de conduzirem a proposições conclusivas. Reconhece-se, aqui, uma falha na metodologia utilizada, passível de ser corrigida em estágio posterior. A única constatação digna de credibilidade, e que corrobora grande parte dos indicativos contidos na literatura, é a que os 
empreendimentos de pequeno porte situados nos setores tradicionais da economia tendem a implementar, com maior intensidade, inovações incrementais.

Também os dados relativos aos perfis dos usuários do sistema confirmam os apontamentos da literatura, sobretudo no que diz respeito às resistências oferecidas pelos empreendimentos de pequeno porte à realização de investimentos em inovações. Trata-se, contudo, de conclusão que deve ser tratada com limites, demandando, pois, investigação posterior mais aprofundada.

A última conclusão digna de destaque aponta para: de um lado, as virtudes do sistema SBRT como gestor de conhecimentos e provedor de respostas técnicas com potencial de gerarem inovações de baixa complexidade em empreendimentos de pequeno porte; de outro lado, as virtudes da estratégia operacional particular do SIRT como agente, não apenas de respostas técnicas, mas com potencial de atuar como agente de inovações.

\section{REFERÊNCIAS}

Amato Neto, J., \& Olave, M. E. L. (2005). A formação de redes de cooperação e clusters em países emergentes: uma alternativa para PMEs no Brasil. In: J. AMATO NETO (Org.), Redes entre organizações: domínio do conhecimento e da eficácia operacional (1st ed., pp. 68 - 93). São Paulo: Atlas.

Barge-Gil, A. (2010). Cooperation-based innovators and peripheral cooperators: an empirical analysis of their characteristics and behavior. Technovation, 30(3), 195-206. Retrieved from: <http://doi.org/10.1016/j.technovation.2009.11.004>.

Bodas Freitas, I. M., Geuna, A., \& Rossi, F. (2013). Finding the right partners: institutional and personal modes of governance of university-industry interactions. Research Policy, 42(1), 50-62. Retrieved from: <http://doi.org/10.1016/j.respol.2012.06.007>.

Castelli, P. G., \& Wilkinson, J. (2013, December 8). Conhecimento tradicional, inovação e direitos de proteção. Estudos, Sociedade e Agricultura. Retrieved from: 〈http://r1.ufrrj.br/esa/V2/ojs/index.php/esa/article/view/221>.

Chesbrough, H. W. (2003). Open innovation: the new imperative for creating and profiting from technology (1st ed.). Boston: Harvard Business School Press.

D'Este, P., Iammarino, S., Savona, M., \& von Tunzelmann, N. (2012). What hampers innovation? Revealed barriers versus deterring barriers. Research Policy, 41(2), 482-488. Retrieved from: <http://doi.org/10.1016/j.respol.2011.09.008>.

Doh, S., \& Kim, B. (2014). Government support for SME innovations in the regional industries: The case of government financial support program in South Korea. Research Policy, 43(9), 1557-1569. Retrieved from: 〈http://doi.org/10.1016/j.respol.2014.05.001>. 
Inovação em micro e pequenas empresas por meio do serviço brasileiro de respostas técnicas

Fonseca, S. A., \& Barboza, R. A. B. (2014). Relatório de atividades sistema integrado de respostas técnicas universidade estadual paulista (UNESP). Araraquara. Retrieved from: $<$ https://www.google.com.br/url?sa=t\&rct=j\&q=\&esrc=s\&source=web\&cd=1\&cad=rja\&uact=8\&v ed=0CB4QFjAA\&url=http://brasilproximocentropaulist.com.br/download.php?id=5aa429f5f59d79 efcea597b6e06f885c\&type=2\&ei=MAlNVaCKCIuxggT3zYHoCg\&usg=AFQjCNFfq8XdinDKdP BsAYGKyq61>.

Freel, M. S. (2000). Barriers to product innovation in small manufacturing firms. International Small Business Journal, 18(2), 60-80. Retrieved from: 〈http://doi.org/10.1177/0266242600182003〉.

Grimpe, C., \& Sofka, W. (2009). Search patterns and absorptive capacity: Low- and high-technology sectors in European countries. Research Policy, 38(3), 495-506. Retrieved from: <http://doi.org/10.1016/j.respol.2008.10.006>.

Heidenreich, M. (2009). Innovation patterns and location of European low- and medium-technology industries. Research Policy, 38(3), 483-494. Retrieved from: <http://doi.org/10.1016/j.respol.2008.10.005>.

Huizingh, E. K. R. E. (2011). Open innovation: State of the art and future perspectives. Technovation, 31(1), 2-9. Retrieved from: 〈http://doi.org/10.1016/j.technovation.2010.10.002〉.

Kalar, B., \& Antoncic, B. (2015). The entrepreneurial university, academic activities and technology and knowledge transfer in four European countries. Technovation, 36-37, 1-11. Retrieved from: <http://doi.org/10.1016/j.technovation.2014.11.002>.

Kirner, E., Kinkel, S., \& Jaeger, A. (2009). Innovation paths and the innovation performance of lowtechnology firms-An empirical analysis of German industry. Research Policy, 38(3), 447-458. Retrieved from: 〈http://doi.org/10.1016/j.respol.2008.10.011>.

Landry, R., Amara, N., Cloutier, J.-S., \& Halilem, N. (2013). Technology transfer organizations: services and business models. Technovation, 33(12), 431-449. Retrieved from: <http://doi.org/10.1016/j.technovation.2013.09.008>.

Love, J. H., Roper, S., \& Vahter, P. (2014). Dynamic complementarities in innovation strategies. Research Policy, 43(10), 1774-1784. Retrieved from: <http://doi.org/10.1016/j.respol.2014.05.005>.

McGuirk, H., Lenihan, H., \& Hart, M. (2015). Measuring the impact of innovative human capital on small firms' propensity to innovate. Research Policy, 44(4), 965-976. Retrieved from: $<$ http://doi.org/10.1016/j.respol.2014.11.008>.

McKelvey, M., Zaring, O., \& Ljungberg, D. (2014). Creating innovative opportunities through research collaboration: An evolutionary framework and empirical illustration in engineering. Technovation, 39, 26-36. Retrieved from: 〈http://doi.org/10.1016/j.technovation.2014.05.008>.

Minayo, M. C. de S., Assis, S. G. de, \& Souza, E. R. de. (2014). Avaliação por triangulação de métodos: Abordagem de Programas Sociais (4th ed.). Rio de Janeiro: Editora Fio Cruz.

Mundt, M., \& Bignetti, L. P. (1992). Apoio tecnológico a empresas de pequeno porte. São Paulo: USP/FEA/IA/PACTo. 
Nunes, P. M., Serrasqueiro, Z., \& Leitão, J. (2012). Is there a linear relationship between R\&D intensity and growth? Empirical evidence of non-high-tech vs. high-tech SMEs. Research Policy, 41(1), 36-53. Retrieved from: 〈http://doi.org/10.1016/j.respol.2011.08.011.>

Oakey, R., Rothwell, R., \& Coope, S. (1988). The management of innovation in high-technology small firms: innovation and regional development in Britain and the United States (1st ed.). New York: Quorum Books.

Quandt, C. O. (2012, April 24). Redes de cooperação e inovação localizada: estudo de caso de um arranjo produtivo local. RAI: revista de administração $e$ inovação. Retrieved from: <http://www.revistas.usp.br/rai/article/view/79254>.

Radas, S., \& Božić, L. (2009). The antecedents of SME innovativeness in an emerging transition economy. Technovation, 29(6-7), 438-450. Retrieved from: <http://doi.org/10.1016/j.technovation.2008.12.002>.

Schumpeter, J. A. (1934). Teoria do desenvolvimento econômico: um estudo sobre lucro empresarial, capital, credito, juro e ciclo da conjuntura. Harvard University Press (1st ed.).

Schumpeter, J. A. (1961). Capitalismo, socialismo e democracia. Rio de Janeiro: Editora Fundo de Cultura S.A.

Sebrae. (2015). Pequenos negócios em números. Brasília. Retrieved from: <http://www.sebraesp.com.br/index.php/234-uncategorised/institucional/pesquisas-sobre-micro-epequenas-empresas-paulistas/micro-e-pequenas-empresas-em-numeros $>$.

Selltiz, C., Wrightsman, L., Cook, S., \& Kidder, L. (1987). Métodos de Pesquisa nas Relações Sociais. São Paulo: EPU - Editora Pedagógica e Universitária Ltda.

Solomon, G. T., Bryant, A., May, K., \& Perry, V. (2013). Survival of the fittest: Technical assistance, survival and growth of small businesses and implications for public policy. Technovation, 33(8-9), 292-301. Retrieved from: 〈http://doi.org/10.1016/j.technovation.2013.06.002>.

Spithoven, A., Clarysse, B., \& Knockaert, M. (2011). Building absorptive capacity to organise inbound open innovation in traditional industries. Technovation, 31(1), 10-21. Retrieved from: <http://doi.org/10.1016/j.technovation.2010.10.003>.

Theodorakopoulos, N., Snchez Preciado, D. J., \& Bennett, D. (2012). Transferring technology from university to rural industry within a developing economy context: The case for nurturing communities of practice. Technovation, 32(9-10), 550-559. Retrieved from: <http://doi.org/10.1016/j.technovation.2012.05.001>.

Tödtling, F., Lehner, P., \& Kaufmann, A. (2009). Do different types of innovation rely on specific kinds of knowledge interactions? Technovation, 29(1), 59-71. Retrieved from: <http://doi.org/10.1016/j.technovation.2008.05.002>.

Velu, C. (2014). Business model innovation and third-party alliance on the survival of new firms. Technovation, 35, 1-11. Retrieved from: 〈http://doi.org/10.1016/j.technovation.2014.09.007〉.

Vieira Filho, J. E. R., \& Silveira, J. M. F. J. da. (2012). Mudança tecnológica na agricultura: uma revisão crítica da literatura e o papel das economias de aprendizado. Revista de Economia $e$ 
Inovação em micro e pequenas empresas por meio do serviço brasileiro de respostas técnicas

Sociologia Rural, 50(4), 721-742. Retrieved from: <http://doi.org/10.1590/S010320032012000400008.>

Vrgovic, P., Vidicki, P., Glassman, B., \& Walton, A. (2014). Open innovation for SMEs in developing countries - An intermediated communication network model for collaboration beyond obstacles. Retrieved from: < http://www.tandfonline.com/doi/abs/10.5172/impp.2012.14.3.290\#.VS7CcdzF$\underline{\mathrm{VM}}>$.

Yin, R. K. (2010). Estudo de caso: planejamento e métodos. Porto Alegre: BOOKMAN.

\title{
INNOVATION IN MICRO AND SMALL ENTERPRISES THROUGH THE SERVICE BRAZILIAN TECHNICAL ANSWERS
}

\begin{abstract}
This paper reports the results of a research which had two main objectives: the first one, to outline the profile in it's institutional, structural and operational aspects of a national system organized for small enterprises (SBRT), organized in the form of national network of agents academic, technological and representative of the business community; and the second (and main) draw a parallel between the predominant strategy of SBRT and strategy, alternative and innovative, adopted by a one of agents. The research, qualitative, exploratory and designed as survey study, revealed that the central strategy of the network is to attend, in the format of technical notes, the spontaneous demands posted in the system. In the other hand, the institution whose activities were assessed sought to differentiate it's activities, adopting a strategy of inducing demands, which goes beyond the simple preparation of technical notes and begins to act as an effective agent of innovations.
\end{abstract}

Keywords: Technical notes; Low complexity innovations; Small firms; Knowledge management; Innovation management.

Data do recebimento do artigo: 02/05/2014

Data do aceite de publicação: 05/06/2015

Revista de Administração e Inovação, São Paulo, v. 12, n.3 p. 330-349, jul./set. 2015. 